\title{
Observation of a Large Atomic Parity Violation Effect in Ytterbium
}

\author{
K. Tsigutkin, ${ }^{1, *}$ D. Dounas-Frazer, ${ }^{1}$ A. Family, ${ }^{1}$ J.E. Stalnaker, ${ }^{1, \dagger}$ V. V. Yashchuk, ${ }^{2}$ and D. Budker ${ }^{1,3}$ \\ ${ }^{1}$ Department of Physics, University of California at Berkeley, Berkeley, California 94720-7300, USA \\ ${ }^{2}$ Advanced Light Source Division, Lawrence Berkeley National Laboratory, Berkeley California 94720, USA \\ ${ }^{3}$ Nuclear Science Division, Lawrence Berkeley National Laboratory, Berkeley, California 94720, USA
}

(Received 19 June 2009; published 10 August 2009)

\begin{abstract}
Atomic parity violation has been observed in the $6 s^{2}{ }^{1} S_{0} \rightarrow 5 d 6 s^{3} D_{1} 408$-nm forbidden transition of ytterbium. The parity-violating amplitude is found to be 2 orders of magnitude larger than in cesium, where the most precise experiments to date have been performed. This is in accordance with theoretical predictions and constitutes the largest atomic parity-violating amplitude yet observed. This also opens the way to future measurements of neutron distributions and anapole moments by comparing parity-violating amplitudes for various isotopes and hyperfine components of the transition.
\end{abstract}

PACS numbers: 11.30.Er, 32.90.+a

Atomic parity violation (APV) experiments are a powerful tool in the study of electroweak interactions (see, for example, review [1]). The electroweak parameter of utmost importance in APV experiments is the weak charge $Q_{W}$, associated with the exchange of the $Z_{0}$ boson between an atomic electron and the nucleus. The most accurate APV experiments were performed using Cs atomic beam and yielded a value of the $Q_{W}$ for Cs with experimental and theoretical uncertainties of $0.35 \%$ [2] and $0.27 \%$ [3], respectively, providing a stringent test of the standard model $(\mathrm{SM})$ at low momentum transfer $(\approx \mathrm{MeV} / c)$. However, it has not yet been possible to test an important prediction of the SM concerning the variation of $Q_{W}$ along a chain of isotopes. It has been suggested [4] that rare-earth atoms may be good candidates for APV experiments because they have chains of stable isotopes, and the APV effects may be enhanced due to the proximity of opposite-parity levels. While the accuracy of atomic calculations is unlikely to ever approach that achieved for atoms with a single valence electron, ratios of PV amplitudes between different isotopes should provide ratios of weak charges, without involving, to first approximation, any atomic-structure calculations.

The present experiment is inspired by the prediction [5] supported by further theoretical work of $[6,7]$, that the PV amplitude in the chosen transition is $\approx 100$ times larger than that in Cs. The motivation for PV experiments in $\mathrm{Yb}$ is probing low-energy nuclear physics by comparing $\mathrm{PV}$ effects on either a chain of naturally occurring $\mathrm{Yb}$ isotopes, or in different hyperfine components for the same odd-neutron-number isotope. The ratio of PV amplitudes for two isotopes of the same element is sensitive to the neutron distributions within the nucleus [8]. The difference between PV amplitudes measured on two different hyperfine lines belonging to the same transition is a manifestation of nuclear-spin-dependent APV, which is sensitive to the nuclear anapole moments (see, for example, reviews $[9,10]$ ) that arise from weak interactions between the nucleons. As the precision of the experiment increases, a sensitive test of the standard model may also become possible [11].

Here we report on experimental verification of the predicted $\mathrm{PV}$-amplitude enhancement in $\mathrm{Yb}$ using a measurement of the APV amplitude for ${ }^{174} \mathrm{Yb}$.

The idea of the experiment is to excite the forbidden 408-nm transition (Fig. 1) with resonant laser light in the presence of a quasistatic electric field. The PV amplitude of this transition arises due to PV mixing of the $5 d 6 s^{3} D_{1}$ and $6 s 6{ }^{1} P_{1}$ states. The purpose of the electric field is to provide a reference transition amplitude due to Stark mixing of the same states, interfering with the PV amplitude. In such interference method [12,13], one is measuring the part of the transition probability that is linear in both the reference Stark-induced amplitude and the PV amplitude. In addition to enhancing the PVdependent signal, employing the Stark-PV-interference technique provides for all-important reversals allowing one to separate the PV effects from various systematics.

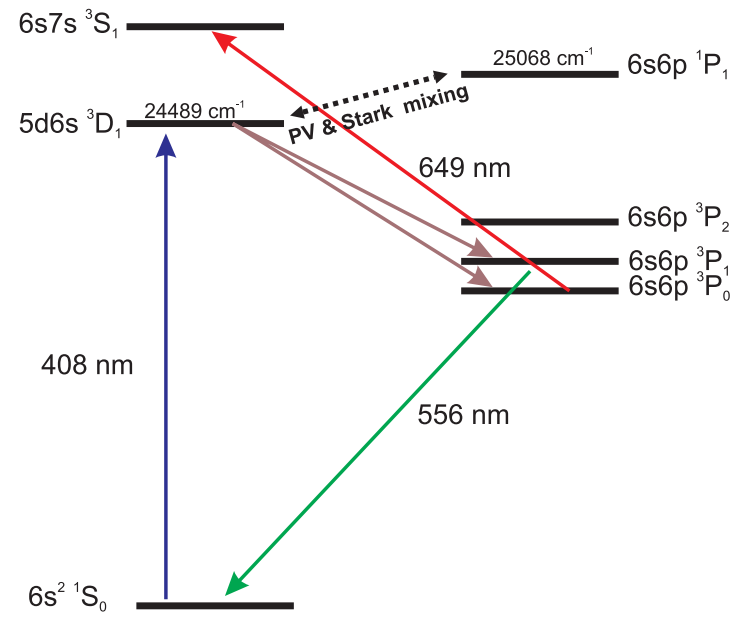

FIG. 1 (color online). Low-lying energy eigenstates of $\mathrm{Yb}$ and transitions relevant to the APV experiment. 
The configuration which is used for the Starkinterference experiment is shown in Fig. 2. The electric field $\mathbf{E}$ is applied collinearly with the propagation axis ( $\mathbf{x})$ of the linearly polarized resonant light beam, while the magnetic field $\mathbf{B}$ is directed along $\mathbf{z}$. The pseudoscalar quantity which manifests $\mathrm{PV}$ is

$$
(\mathcal{E} \cdot \mathbf{B})([\mathbf{E} \times \mathcal{E}] \cdot \mathbf{B}),
$$

where $\mathcal{E}$ is the electric field of the light. The APV effect vanishes when the angle $\theta$ between the light polarization and the magnetic field approaches a value which is a multiple of $\pi / 2$.

This field arrangement is such that the $M 1$ transition amplitude and Stark-induced amplitudes are out of phase [14]. Thus, the $M 1$ Stark interference is suppressed. Additional suppression is provided by the use of a power-build-up cavity. The $M 1$ transition amplitude proportional to $\mathbf{k} \times \mathcal{E}$ vanishes to the degree that the field in the cavity is a standing wave, and the net wave vector $\mathbf{k}$ is suppressed.

For an isotope with zero nuclear spin $I$, there are three Zeeman-split components of the transition. A Starkinduced transition amplitude is generally expressed in terms of real scalar $(\alpha)$, vector $(\beta)$, and tensor $(\gamma)$ transition polarizabilities $[12,15]$; however, for the case of a $J=0 \rightarrow J^{\prime}=1$ transition, only the vector transition polarizability contributes. Assuming that the magnetic field is strong enough to resolve the Zeeman components of the transition and selecting the quantization axis along the magnetic field, we obtain the following transition rates:

$$
\begin{aligned}
\mathcal{R}_{\Delta M=0} & =\frac{8 \pi}{c} I\left[\beta^{2} E^{2} \sin ^{2} \theta+2 \zeta \beta E \sin \theta \cos \theta\right], \\
\mathcal{R}_{\Delta M= \pm 1} & =\frac{4 \pi}{c} I\left[\beta^{2} E^{2} \cos ^{2} \theta-2 \zeta \beta E \sin \theta \cos \theta\right],
\end{aligned}
$$

where $I$ is the light intensity. Here $\zeta$ characterizes the PV-

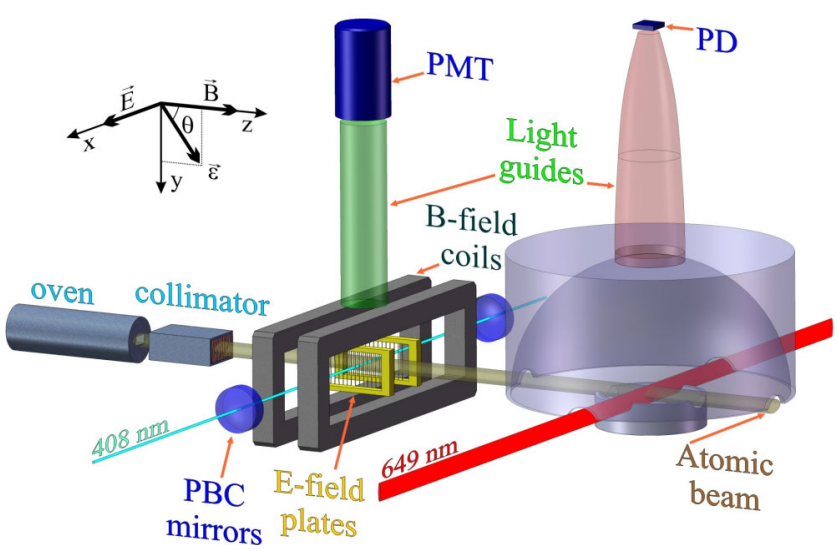

FIG. 2 (color online). Orientation of fields for PV-Stark interference experiment and schematic of the present APV apparatus. Not shown is the vacuum chamber containing all the depicted elements, except the photomultiplier (PMT) and the photodiode (PD). PBC-power buildup cavity. Light is applied collinearly with $\mathbf{x}$. induced electric-dipole transition amplitude between states with total angular momenta and projections $F, M$ and $F^{\prime}$, $M^{\prime}$ :

$$
A_{F M F^{\prime} M^{\prime}}^{\mathrm{PV}}=i \zeta_{F F^{\prime}}(-1)^{q} \mathcal{E}_{q}\left\langle F, M, 1,-q \mid F^{\prime}, M^{\prime}\right\rangle,
$$

where $q=M-M^{\prime}$ labels the spherical component and $\left\langle F, M, 1,-q \mid F^{\prime}, M^{\prime}\right\rangle$ is a Clebsch-Gordan coefficient. In expressions (2) and (3), we neglect the term quadratic in PV mixing. Using the theoretical value of $\zeta \simeq 10^{-9} e a_{0}$ [6] combined with the measured $|\beta|=2.24_{-0.12}^{+0.07} \times$ $10^{-8} e a_{0} /(\mathrm{V} / \mathrm{cm})[15,16]$, the expected relative strength of the PV effect, $2 \zeta / \beta E$, is $\sim 10^{-4}$, for $\theta=\pi / 4$ and $E=$ $1 \mathrm{kV} / \mathrm{cm}$.

The transition rates (2) and (3), are detected by measuring the population of the $6 s 6 p^{3} P_{0}$ state, where $65 \%$ of the atoms excited to the $5 d 6 s^{3} D_{1}$ state decay spontaneously (Fig. 1). This is done by resonantly exciting the atoms with 649-nm light to the $6 s 7 s^{3} S_{1}$ state downstream from the main interaction region and collecting the fluorescence resulting from the decay of this state back to $6 s 6 p^{3} P_{0}$ state, and also to $6 s 6 p^{3} P_{1}$ and $6 s 6 p^{3} P_{2}$ states. As long as the 408-nm transition is not saturated, the fluorescence intensity measured in the probe region is proportional to the rate of that transition.

In order to isolate the Stark-PV interference term in the transition rate from the dominant Stark-induced transition rate, we harmonically modulate the applied electric field. The dominant Stark-induced rate has a static component and a component oscillating at twice the modulation frequency, while the Stark-PV interference term oscillates at the first harmonic. The frequency discrimination is performed using lock-in amplifiers. For an arbitrary angle of the light polarization $\theta$, there are generally three Zeeman components of the transition present while scanning over the profile as shown in Fig. 3(a). The first-harmonic signal due to Stark-PV interference has a characteristic signature: the sign of the phase of the modulation of the two extreme components of the transition is opposite to that of the central component. The second-harmonic signal provides a reference for the line shape since it is free from interference effects linear in $\mathbf{E}$ [Fig. 3(b)]. If, in addition to the oscillating electric field, there is also a dc component present in the applied field, a signature identical to that in the second harmonic will also appear in the first harmonic, Fig. 3(c). The latter can be used to increase the first-harmonic signal above the noise, which makes the profile analysis more reliable.

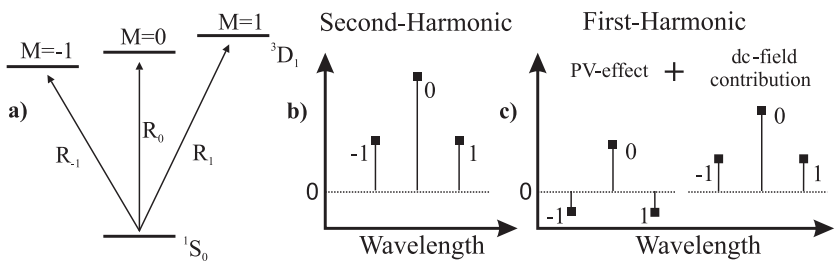

FIG. 3. Discrimination of the PV effect by $E$-field modulation under static magnetic field. 
A schematic of the Yb-APV apparatus is shown in Fig. 2. $\mathrm{A}$ beam of $\mathrm{Yb}$ atoms is produced (inside of a vacuum chamber with a residual pressure of $\approx 3 \times 10^{-6}$ Torr) with an effusive source, which is a stainless-steel oven loaded with $\mathrm{Yb}$ metal, operating at $500{ }^{\circ} \mathrm{C}$. The oven is outfitted with a multislit nozzle, and there is an external vane collimator reducing the spread of the atomic beam in the horizontal direction. The resulting Doppler width of the 408-nm transition is $\approx 12 \mathrm{MHz}$ [16].

Downstream from the collimator, the atoms enter the main interaction region where the Stark- and PV-induced transitions take place. Up to $80 \mathrm{~mW}$ of light at the transition wavelength of $408.345 \mathrm{~nm}$ in vacuum is produced by frequency doubling the output of a Ti:sapphire laser (Coherent 899).

The 408-nm light is coupled into a power buildup cavity (PBC) inside the vacuum chamber. The finesse and the circulating power of the PBC are measured to be up to $\mathcal{F}=9000$ and $P=8 \mathrm{~W}$, respectively. The laser is locked to the PBC using the FM-sideband technique [17]. In order to remove frequency excursions of the $\mathrm{PBC}$ in the acoustic range, the cavity is locked to a more stable confocal FabryPérot étalon, once again using the FM-sideband technique. This stable scannable cavity provides the master frequency, with the power-build-up cavity serving as the secondary master for the laser.

The magnetic field is generated by a pair of rectangular coils designed to produce a uniform magnetic field up to $100 \mathrm{G}: 1 \%$ nonuniformity over the volume with the dimensions of $1 \times 1 \times 1 \mathrm{~cm}^{3}$ in the interaction region. Additional coils placed outside of the vacuum chamber compensate for the external magnetic fields down to $10 \mathrm{mG}$ at the interaction region. The residual $B$ field of this magnitude does not have an impact on the PV-effect measurements, since its contribution is measured using the field reversals (see below).

The electric field is generated with two wire-frame electrodes separated by $2 \mathrm{~cm}$. The copper electrode frames support arrays of $0.2-\mathrm{mm}$ diameter gold-plated wires. This design allows us to reduce the stray charges accumulated on the electrodes by minimizing the surface area facing the atomic beam, thus minimizing stray electric fields. ac voltage up to $10 \mathrm{kV}$ at a frequency of $76.2 \mathrm{~Hz}$ is supplied to the $E$-field electrodes by a homebuilt high-voltage amplifier. An additional dc bias voltage up to $100 \mathrm{~V}$ can be added.

Light emitted from the interaction region at $556 \mathrm{~nm}$ is collected with a light guide and detected with a photomultiplier tube. This signal is used for initial selection of the atomic resonance and for monitoring purposes. Fluorescent light from the probe region is collected onto a light guide using two optically polished curved aluminum reflectors and registered with a cooled photodetector (PD). The PD consists of a large-area $\left(1 \times 1 \mathrm{~cm}^{2}\right)$ Hamamatsu photodiode connected to a $1-G \Omega$ transimpedance preamplifier, both contained in a cooled housing (temperatures down to $-15^{\circ} \mathrm{C}$ ). The preamp's bandwidth is $1 \mathrm{kHz}$ and the output noise is $\sim 1 \mathrm{mV}$ (rms). The 649-nm excitation light is derived from a single-frequency diode laser (New Focus Vortex) producing $\approx 1.2 \mathrm{~mW}$ of $\mathrm{cW}$ output, high enough to saturate the $6 s 6 p^{3} P_{0} \rightarrow 6 s 7 s^{3} S_{1}$ transition. A drift of the laser frequency is eliminated by locking the diode laser to a transfer cavity, in turn locked to a frequency-stabilized He-Ne laser.

The signals from the PMT and PD are fed into lock-in amplifiers for frequency discrimination and averaging. The typical time of a single spectral profile acquisition is $20 \mathrm{~s}$. The first- and second-harmonic signals are registered simultaneously, which reduces the influence of slow drifts, such as instabilities of the atomic-beam flux. The modulation frequency is limited by several factors. Thermal distribution of atomic velocities in the beam causes a spread (of $\approx 2 \mathrm{~ms}$ ) in the time of flight between the interaction region and the probe region. This, along with the finite bandwidth of the PD, leads to a reduction of the signalmodulation contrast. The choice of the modulation frequency of $76.2 \mathrm{~Hz}$ is a tradeoff between this contrast degradation and the requirement of lock-in detection over many modulation periods.

In Fig. 4 a profile of the $B$-field-split 408-nm spectral line of the ${ }^{174} \mathrm{Yb}$ isotope is shown. The 649-nm lightinduced fluorescence was recorded during a typical integration run. Statistical error bars are smaller than the size of the points in the figure. The peculiar asymmetric line shape of the Zeeman components is a result of the dynamic Stark effect [16]. The profiles are fit to an approximated analytic function. The fit amplitudes of the three peaks yield the fluorescence amplitudes for the different Zeeman components at 1 st and 2 nd harmonics of the modulation. Ideally, in the absence of apparatus imperfections and systematic effects, the following combination of amplitude ratios between the 1st and 2nd harmonics yields the PVinterference effect:

$$
\mathcal{K}=\frac{\mathcal{A}_{-1}^{1 \mathrm{st}}}{\mathcal{A}_{-1}^{2 \mathrm{nd}}}+\frac{\mathcal{A}_{+1}^{1 \mathrm{st}}}{\mathcal{A}_{+1}^{2 \mathrm{nd}}}-2 \frac{\mathcal{A}_{0}^{1 \mathrm{st}}}{\mathcal{A}_{0}^{2 \mathrm{nd}}}=\frac{16 \zeta}{\beta \tilde{E}}
$$
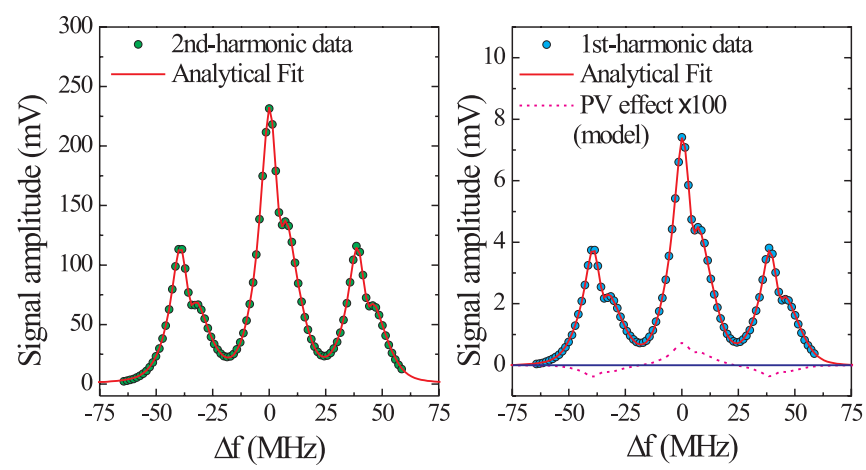

FIG. 4 (color online). A profile of the $B$-field-split $408-\mathrm{nm}$ spectral line of ${ }^{174} \mathrm{Yb}$ recorded at $1 \mathrm{st}$ and 2 nd harmonic of the modulation. Also a simulated PV contribution is shown for clarity. $\tilde{E}=5 \mathrm{kV} / \mathrm{cm}$; dc offset $=40 \mathrm{~V} / \mathrm{cm} ; \theta=\pi / 4$; an effective integration time is $10 \mathrm{~s}$ per point. 


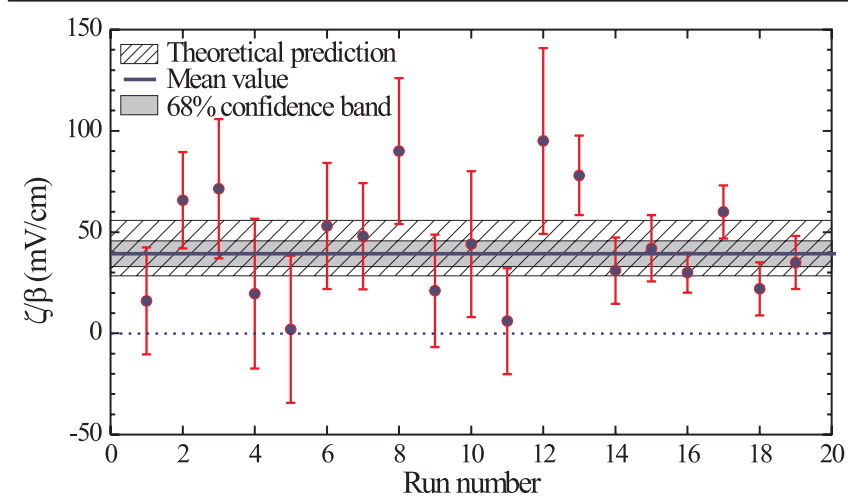

FIG. 5 (color online). The PV-interference parameter $\zeta / \beta$. Mean value: $39(4)_{\text {stat }}(5)_{\text {syst }} \mathrm{mV} / \mathrm{cm},|\zeta|=8.7 \pm 1.4 \times 10^{-10} e a_{0}$. The $68 \%$ confidence band includes both the statistical and the systematic uncertainties.

where $\mathcal{A}_{ \pm 1}, \mathcal{A}_{0}$ are the amplitudes of the respective Zeeman components and $\tilde{E}$ is the amplitude of modulating electric field.

The detailed analysis of an impact of the apparatus imperfections and systematic effects on the accuracy of the measurements will be presented elsewhere. Here we address briefly the principles of this analysis. The PV effect is discriminated from other effects by recording the spectral profiles for different combinations of the $B$ field and the light polarization angle $\theta$, and by isolating the part of the measured values of $\mathcal{K}$ that has a correct $\mathrm{PV}$ response upon the reversals. In addition we artificially impose exaggerated combinations of imperfections and measure their effect on $\mathcal{K}$. Then, by scaling down these contributions we estimate the residual uncertainties in the PV measurements. Such experiments showed a negligibly small contribution of the imperfections compared to the present accuracy of the PV-effect determination (see below).

In Fig. 5, the PV-interference parameter $\zeta / \beta$ is shown as determined in 19 consecutive runs ( $\sim 60 \mathrm{~h}$ of integration). Its mean value is $39(4)_{\text {stat }}(5)_{\text {syst }} \mathrm{mV} / \mathrm{cm}$, which is in agreement with the theoretical predictions. Thus, $|\zeta|=8.7 \pm$ $1.4 \times 10^{-10} e a_{0}$, which is the largest APV amplitude observed so far. This confirms the predicted enhancement of the PV effect in $\mathrm{Yb}$.

The present measurement accuracy is not yet sufficient to observe the isotopic and hyperfine differences in the $\mathrm{PV}$ amplitude. It must be better than $\approx 1 \%$ for $\mathrm{PV}$ amplitude in a single transition $[11,18,19]$. We found that the main factors limiting the present accuracy are fluctuations of the electric field in the interaction region (due to stray fields and HV-amplifier noises), and frequency excursions of the Fabry-Pérot étalon serving as a frequency reference for the optical system. A direct impact of these factors on the spectral profiles has been observed, thus leading to errors not only in the APV measurements, but also in the study of systematic effects and apparatus imperfections. This accounts for the relatively large systematic uncertainty of the
PV parameter. In the course of the APV measurements, several improvements have been implemented targeting these noise sources. They have demonstrated a possibility to reduce the measurement errors substantially. This is seen in Fig. 5, where the last six measurements exhibit higher accuracy than the rest. An upgrade of the apparatus is under way aimed at eliminating the noise sources, which will open ways to the measurements of neutron distributions and anapole moments.

The authors acknowledge helpful discussions with and important contributions of M. A. Bouchiat, C. J. Bowers, E. D. Commins, B.P. Das, D. DeMille, A. Dilip, S.J. Freedman, J.S. Guzman, G. Gwinner, D.F. Kimball, M. G. Kozlov, S. M. Rochester, and M. Zolotorev. This work has been supported by NSF.

*tsigutkin@berkeley.edu

${ }^{\dagger}$ Present address: Department of Physics and Astronomy, Oberlin College, Oberlin, OH 44074, USA.

[1] J. Guena, M. Lintz, and M. A. Bouchiat, Mod. Phys. Lett. A 20, 375 (2005).

[2] C.S. Wood, S. C. Bennett, D. Cho, B. P. Masterson, J. L. Roberts, C.E. Tanner, and C.E. Wieman, Science 275, 1759 (1997).

[3] S. G. Porsev, K. Beloy, and A. Derevianko, Phys. Rev. Lett. 102, 181601 (2009).

[4] V.A. Dzuba, V. V. Flambaum, and I. B. Khriplovich, Z. Phys. D 1, 243 (1986).

[5] D. DeMille, Phys. Rev. Lett. 74, 4165 (1995).

[6] S. G. Porsev, G. Rakhlina Yu, and M. G. Kozlov, JETP Lett. 61, 459 (1995).

[7] B. P. Das, Phys. Rev. A 56, 1635 (1997).

[8] E. N. Fortson, Y. Pang, and L. Wilets, Phys. Rev. Lett. 65, 2857 (1990).

[9] J.S. M. Ginges and V. Flambaum, Phys. Rep. 397, 63 (2004).

[10] W. C. Haxton and C. E. Wieman, Annu. Rev. Nucl. Part. Sci. 51, 261 (2001).

[11] B. A. Brown, A. Derevianko, and V. V. Flambaum, Phys. Rev. C 79, 035501 (2009).

[12] M. A. Bouchiat and C. Bouchiat, J. Phys. II (France) 36, 493 (1975).

[13] R. Conti, P. Bucksbaum, S. Chu, E. D. Commins, and L. Hunter, Phys. Rev. Lett. 42, 343 (1979).

[14] P.S. Drell and E. D. Commins, Phys. Rev. A 32, 2196 (1985).

[15] C. J. Bowers, D. Budker, S. J. Freedman, G. Gwinner, J. E. Stalnaker, and D. DeMille, Phys. Rev. A 59, 3513 (1999).

[16] J. E. Stalnaker, D. Budker, S. J. Freedman, J. S. Guzman, S. M. Rochester, and V. V. Yashchuk, Phys. Rev. A 73, 043416 (2006).

[17] R. W. P. Drever, J. L. Hall, F. V. Kowalski, J. Hough, G. M. Ford, A. J. Munley, and H. Ward, Appl. Phys. B 31, 97 (1983).

[18] S. Porsev, M. Kozlov, and Y. Rakhlina, Hyperfine Interact. 127, 395 (2000).

[19] A. D. Singh and B. P. Das, J. Phys. B 32, 4905 (1999). 